\title{
A rare variant in MCF2L identified using exclusion linkage in a pedigree with premature atherosclerosis
}

\author{
Stephanie Maiwald ${ }^{1,7}$, Mahdi M Motazacker ${ }^{1,7,8}$, Julian C van Capelleveen ${ }^{1}$, Suthesh Sivapalaratnam ${ }^{1}$, \\ Allard C van der Wal ${ }^{2}$, Chris van der Loos ${ }^{2}$, John JP Kastelein ${ }^{1}$, Willem H Ouwehand ${ }^{3,4}$, G Kees Hovingh ${ }^{1}$, \\ Mieke D Trip ${ }^{1,5}$, Jaap D van Buul ${ }^{6}$ and Geesje M Dallinga-Thie ${ }^{\star, 1}$
}

Cardiovascular disease (CVD) is a major cause of death in Western societies. CVD risk is largely genetically determined. The molecular pathology is, however, not elucidated in a large number of families suffering from CVD. We applied exclusion linkage analysis and next-generation sequencing to elucidate the molecular defect underlying premature CVD in a small pedigree, comprising two generations of which six members suffered from premature CVD. A total of three variants showed co-segregation with the disease status in the family. Two of these variants were excluded from further analysis based on the prevalence in replication cohorts, whereas a non-synonymous variant in MCF.2 Cell Line Derived Transforming Sequence-like protein (MCF2L, c.2066A > G; p.(Asp689Gly); NM_001112732.1), located in the DH domain, was only present in the studied family. MCF2L is a guanine exchange factor that potentially links pathways that signal through Rac1 and RhoA. Indeed, in HeLa cells, MCF2L689Gly failed to activate Rac1 as well as RhoA, resulting in impaired stress fiber formation. Moreover, MCF2L protein was found in human atherosclerotic lesions but not in healthy tissue segments. In conclusion, a rare functional variant in MCF2L, leading to impaired DH function, was identified in a small pedigree with premature CVD. The presence of MCF2L in human atherosclerotic plaque specimen lends further support to its potential role in atherosclerosis.

European Journal of Human Genetics (2016) 24, 86-91; doi:10.1038/ejhg.2015.70; published online 22 April 2015

\section{INTRODUCTION}

Cardiovascular Disease (CVD) is the major cause of morbidity and mortality across the globe. In combination with the strong influence of environmental factors, genetic determinants are pivotal in the pathobiology of atherosclerosis, the underlying disease ultimately resulting in CVD events. ${ }^{1}$ This was demonstrated by a $30-60 \%$ heritability in twin studies, and the finding that a positive family history of premature CVD is an independent risk factor. ${ }^{2,3}$ Forty-six common variants with a minor allele frequency $>5 \%$ were found to be associated with CVD in genome-wide association studies in recent years. ${ }^{4,5}$ Collectively, these data showed that only $10 \%$ of CVD risk is attributable to common genetic variations, which supports the hypothesis that low frequency variants may help to explain the observed heritability. ${ }^{4-6}$ Thus, the complex heritability of CVD may be explained by common variants with effect sizes so small that they remain undetected in recent GWAS meta-analysis or by rare variants with an intermediate or large effect. Multi-generation pedigrees with a clear Mendelian form of premature CVD will be extremely instrumental in the identification of these novel pedigree-specific mutations with a large effect on CVD. So far, mutations in Low Density Lipoprotein receptor $(L D L R)$, Low-Density Lipoprotein Receptor Related Protein 6 (LRP6) and Myocyte Enhancer Factor 2 A (MEF2A), Keratocan (KERA), Guanylyl Cyclase $\alpha 1$ subunit (GUCY1A3) and
Chaperonin Containing TCP1, Subunit 7 (CCT7) have been published, although conflicting results have been reported for MEF2A. ${ }^{7-15}$ Collectively, the identification of informative pedigrees with a dominant form of premature CVD have proven to be instrumental in the identification of novel variants in genes involved in the pathobiology of CVD, which may ultimately result in the new therapeutic targets.

The aim of the current study was to identify the molecular defect in a small pedigree of Caucasian descent with an autosomal dominant form of CVD. By combining data from exclusion linkage analysis and next-generation sequencing, we identified a non-synonymous variant located in the DH-domain of MCF2L, a gene encoding for MCF.2 Cell Line Derived Transforming Sequence-like protein also known as the guanine nucleotide exchange factor DBS.

\section{MATERIALS AND METHODS}

Recruitment of the pedigree with early onset CVD

A female subject was referred to the outpatient clinic of the Academic Medical Center (Amsterdam, the Netherlands) for analysis after she suffered from an acute myocardial infarction at the age of 39 years (Figure 1; index II.6). She was a member of a small family with an autosomal dominant form of early onset CVD (Figure 1). A premature CVD event was defined according to the AHA/ ACC criteria as having a documented CVD event before the age of 55 years (male) and 65 years (women). ${ }^{16}$ The affection status was assessed based on medical records and imaging as extensively described in the Supplementary

${ }^{1}$ Departments of Vascular and Experimental Vascular Medicine, Academic Medical Centre, Amsterdam, The Netherlands; ${ }^{2}$ Department of Pathology, Academic Medical Centre, Amsterdam, The Netherlands; ${ }^{3}$ Department of Haematology, University of Cambridge, Cambridge, UK; ${ }^{4}$ National Health service Blood and Transplant, Cambridge, UK; ${ }^{5}$ Department of Cardiology, Academic Medical Centre, Amsterdam, The Netherlands; ${ }^{6}$ Department of Molecular Cell Biology, Sanquin Research and Landsteiner Laboratory, Academic Medical Center, Amsterdam, The Netherlands

*Correspondence: Dr GM Dallinga-Thie, Department of Vascular Medicine, Academic Medical Center, G1.113, meibergdreef 9, Amsterdam 1105AZ, The Netherlands. Tel: +31 20566 1150; Fax: +31 20566 9394; E-mail:g.m.dallinga@amc.nl

${ }^{7}$ These authors contributed equally to this work.

${ }^{8}$ Current address: Department of Genome Diagnostics, AMC.

Received 4 September 2014; revised 27 February 2015; accepted 10 March 2015; published online 22 April 2015 
a

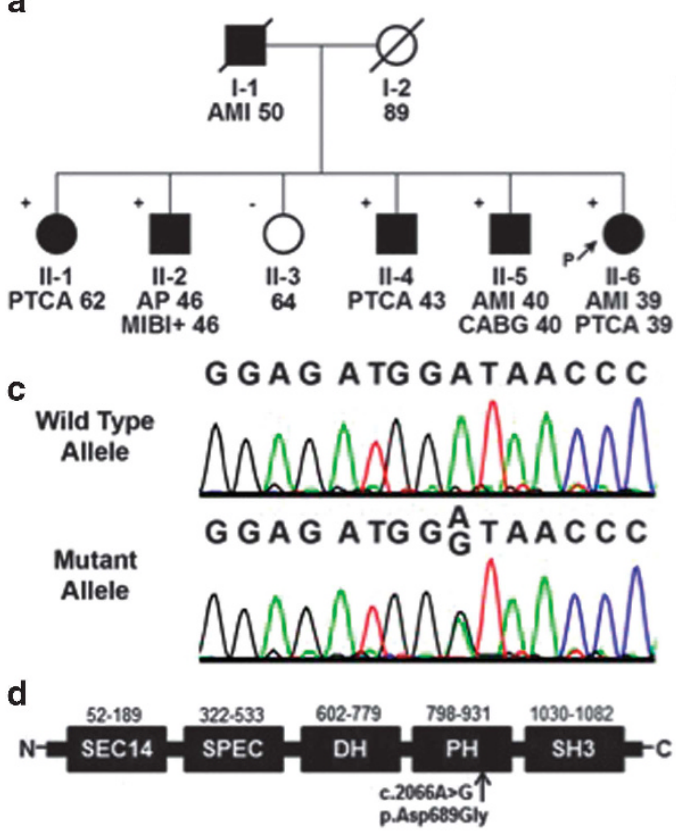

b

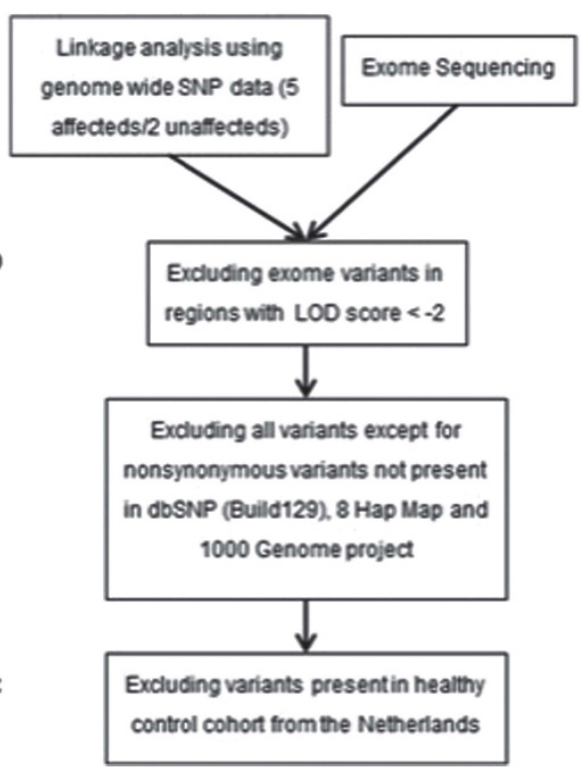

Figure 1 The identification of a mutation in MCF2L. (a) Co-segregation of the MCF2L c.2066A>G p.(Asp689Gly) variant in a small pedigree with premature CVD. Squares represent males and circles represent females. Right half-filled symbols represent cases with premature CVD. The arrow indicated the index case. Left upper forth-part-filled symbols represent heterozygous carriership of the MCF2L c.2066A>G p.(Asp689Gly) variant. The arabic number is an identifier for each individual, whereas the generation is marked with a roman number. DNA of all living 12 individuals was included in the genetic analysis. (b) Schematic overview of the gene-finding strategy. (c) DNA Sanger sequencing chromatogram showing the heterozygote c. 2066 A $>$ G; p.(Asp689Gly) MCF2L variant. (d) Schematic overview of MCF2L (NP_001106203.2, 1125 amino acids, molecular weight approximately 128 Da), indicating the DHdomain (guanine nucleotide exchange factor) and the PH-domain (DBL's big sister). At the N-terminus, a Sec14 (Sec14p-like lipid binding domain) and Spectrin repeats (SPEC) are located. At the C-terminus, a SH3 (Src homology 3 domain of DBL's big sister) is present.

Methods (Supplementary Table S1). The study complies with the Declaration of Helsinki and the Institutional Review Board of the AMC of the University of Amsterdam approved the study and all participants provided written informed consent.

Blood was collected from the index case and her relatives, after an overnight fast, in EDTA-coated tubes. Plasma was isolated after centrifugation at $1600 \mathrm{~g}$, $4{ }^{\circ} \mathrm{C}$ for $20 \mathrm{~min}$ and stored at $-80^{\circ} \mathrm{C}$ for further analyses. Plasma cholesterol, low-density lipoprotein-cholesterol, high-density lipoprotein cholesterol and triglycerides were analyzed using commercially available assays (Randox, Antrim, United Kingdom and Wako, Neuss, Germany) on a Cobas-Mira autoanalyzer (Roche, Basel, Switzerland).

\section{Exclusion linkage analysis and exome sequencing}

Genomic DNA was extracted from whole blood on an Autopure LS system according to the manufacturer's protocol (Gentra Systems, Minneapolis, MN, USA). Human CytoSNP-12 DNA analysis BeadChip kits (Illumina, Eindhoven, the Netherlands) were used for genome-wide single nucleotide polymorphism (SNP) genotyping in five affected and two unaffected relatives (Figure 1) as described previously. ${ }^{17}$ The data quality was checked using Graphical Representation of Relationship errors (GRR) and PedCheck packages. ${ }^{18,19}$ Multipoint parametric linkage analyses was performed using a fully penetrant autosomal dominant model and mutant locus frequency (MAF) of 0.001 using the Allegro program including 110.000 high-quality SNPs throughout the genome..$^{20}$ The maximum theoretical LOD score for linkage analysis in the core pedigree was 1.5. In parallel, exome sequencing using the DNA of the index case was performed using the Agilent SureSelect $38 \mathrm{Mb}$ exome with $>100 \times$ coverage on the Illumina GAII platform (Illumina, Little Chesterford, United Kingdom $)^{17}$ and used for exclusion linkage. ${ }^{21}$ Three missense variants in MCF2L (NM_001112732.1), ZC3HC1 (NM_016478.3) and CAMSAP1 (NM_015447.3) were identified and confirmed with Sanger sequencing as previously described using the following primer pairs: $M C F 2 L$ forward 5'-TGC TTT TGC TTT GAT GGA TG-3' and reverse
5'-CAT TCC AGC CCC CTG AAG-3'; ZC3HC1 forward: $5^{\prime}$-GAG AAA ACT CTC TTT TTC ATT CC-3' and reverse 5'-CAC CCA AAT AAG CTA AGT GAA TAC-3'; CAMSAP1 5'-AAA CAG ATG CTA CCA ATC CCT TAC-3' and reverse $5^{\prime}$-CCT CTT CCA AAG ATG CCA AC- $3{ }^{\prime}{ }^{22}$ The data are registered in the LOVD database under screening number 00027156 (http://databases.lovd.nl/ shared/screenings/0000027156).

\section{Validation cohorts}

Premature AtheroSclerosis cohort. This cohort (Supplementary Table S3) comprises 935 patients with early symptomatic atherosclerosis (CVD) before the age of 51 years. CAD was defined as myocardial infarction, coronary revascularization or evidence of at least $70 \%$ stenosis in a major epicardial artery. ${ }^{23}$ Patients were recruited at the Cardiology and Vascular outpatient clinics of the AMC, Amsterdam, the Netherlands. ${ }^{24}$

Sanquin blood bank common controls. DNA samples were collected from 1440 healthy volunteers who were recruited from a large cohort of blood donors, who were free of CVD, at one of the collection sites of the Sanquin Blood Bank covering the northwest section of the Netherlands, which geographically overlaps the Premature AtheroSclerosis case cohort. ${ }^{24}$

Cambridge bioresource collection. NHS Blood and Transplant enrolled DNA samples of 8946 healthy volunteers in a resource for genotype-phenotype association studies. ${ }^{25}$

\section{MCF2L constructs and cell transfections}

A vector containing human MCF2L (pENTR221/MCF2L; clone IOH23111, Invitrogen, Bleiswijk, the Netherlands) was used. A human MCF2L689Gly vector was generated by site-directed mutagenesis with the Quick QuikChange kit (Stratagene, La Jolla, CA, USA) using the primer pairs: forward 5'-CGC CGC GGA GAT GGG TAA CCC ACT GAT GG-3' and reverse 
5'-GCG GCG CCT CTA CCC ATT GGG TGA CTA CC-3' (QuickChange primer design tool (https://www.genomics.agilent.com). ${ }^{26}$ Sequences were checked with Sanger Sequencing using the following primers: M13 forward 5'-GTT GTA AAA CGA CGG CCA GT-3' and reverse 5'-CAC AGG AAA CAG CTA TGA CC- $3^{\prime}$. Next, the wild-type and mutant constructs were inserted into the destination vector pcDNA-DEST40 (Invitrogen) using the Gateway LR Clonase II enzyme mix (Invitrogen) according to the manufacturer's protocol.

\section{Rac1-GTP pull-down assay}

HeLa cells were cultured in Iscove's Modified Dulbecco's medium (Invitrogen) supplemented with $10 \%(\mathrm{v} / \mathrm{v})$ heat-inactivated fetal calf serum, $1 \%$ glutamine and antibiotics and kept at $37^{\circ} \mathrm{C}$ at $5 \% \mathrm{CO}_{2}$ as previously described. ${ }^{27}$ Cells were transfected with pcDNA-DEST40/MCF2L689Asp or pcDNA-DEST40/ MCF2L689Gly using TransIT-LT1 Transfection Reagent (Mirus, Ochten, the Netherlands). After $24 \mathrm{~h}$ incubation, cells were lysed in RIPA and subsequently centrifugation at $14000 \mathrm{~g}$ for $5 \mathrm{~min}$ was carried out. GTP-bound Racl (Rasrelated botulinum toxin substrate 1) was isolated with biotinylated CRIBpeptide coupled to streptavidin agarose beads during a $30 \mathrm{~min}$ incubation at $4{ }^{\circ} \mathrm{C} .{ }^{28}$ Beads were washed four times in $50 \mathrm{~mm}$ Tris, $\mathrm{pH} 7.4,0.5 \mathrm{~mm} \mathrm{MgCl}_{2}$, $150 \mathrm{~mm} \mathrm{NaCl}, 1 \%(\mathrm{v} / \mathrm{v})$ Triton X-100, supplemented with protease and phosphatase inhibitor cocktails (Roche, Woerden, the Netherlands). Racl was visualized by western blotting using a mouse-anti-human Racl antibody (clone 102, BD bioscience, Breda, the Netherlands).

To image stress fibers, HeLa cells were plated on fibronectin (R\&D systems Europe, Abingdon, United Kingdom)-coated glass cover slips and transfected as described above. Next, cells were fixed with $3.7 \%$ formaldehyde and permeabilized with $0.5 \%$ Triton X-100, stained with rabbit-anti-human MCF2L and visualized with Phalloidin-Texas Red (Invitrogen). F-actin was made visible with ALEXA488-labeled anti-rabbit IgG (Invitrogen). The nuclei were stained with DAPI (Invitrogen). ${ }^{29}$ Images were recorded with LSM510 META microscope (Carl Zeiss Micro Imaging, Inc., Goettingen, Germany)

\section{Human plaque quantification}

Specimens of tonsil $(n=2)$, which is used as a negative control tissue, atherosclerotic artery specimen $(n=9)$ and non-diseased artery specimens $(n=9)$ were collected from patients at autopsy. Coronary arteries were classified according to different stages of atherosclerosis using hematoxylin (Fluka Biochemica, Buchs, Switzerland) and eosin (Sigma Aldrich, St Louis, MO, USA) stains according to standard protocols. Tissue specimens were stained with antibodies against MCF2L (rabbit polyclonal anti-MCF2L, WH0023263M1, Sigma, Munich, Germany), smooth muscle cell $\alpha$-actin (mouse 1A4 antibody, DAKO, Glostrup, Denmark), macrophage CD68 (mouse monoclonal anti-human CD68 PG-M1, DAKO, Heverlee, Belgium), endothelial cell marker CD34 (mouse anti-human CD34Q, Bend10, ThermoFischer Scientific, Waltham, MA, USA), CD11c as marker for antigen-presenting cells (mouse monoclonal 5D11; Monosan, Uden, the Netherlands), CD8 as marker for $\mathrm{T}$ cells (mouse monoclonal anti-human CD8, CD8/144B; DAKO) and CD3 as marker for $\mathrm{T}$ cells (anti-CD3, IgG monoclonal SP7; ThermoFischer Scientific). Secondary antibodies: swine polyclonal anti-rabbit immunoglobulins/HRP 1:3000 (DAKO) and goat polyclonal anti-mouse immunoglobulins/ HRP 1:1000 (DAKO). Additional information is available in the Supplementary Method section.

\section{Statistical analysis}

Results are expressed as mean \pm standard deviation unless otherwise stated. Statistical differences were tested using Students $t$-test statistics. Two-sided $P$ values of $<0.05$ were considered statistically significant. All statistical analyses were performed with SPSS version 18.0 (IBM, Chicago, IL, USA).

\section{RESULTS}

\section{Identification of the mutation in MCF2L}

The index case (Figure 1) suffered from an acute myocardial infarction at the age of 39 years. She had been smoking 20 cigarettes per day before she had the event. Plasma lipids were all within the normal range (Table 1). Upon expansion of the family, it was noted that a total of five relatives did suffer from premature CVD, whereas two relatives were classified as 'unaffected' according to the definition presented in Supplementary Methods (Figure 1a, Supplementary Table S1). Multipoint linkage analysis for exclusion linkage was performed (Supplementary Figure S1, Supplementary Table S2), followed by exome sequencing using DNA from the index case. A total 453100 variants were identified that met the commonly described quality criteria of which 4510 were non-synonomous, indels or splice site variants. ${ }^{17}$ Of these, 961 variants were not found in the large public database (dbSNP129), 8 Hapmap project exomes ${ }^{30}$ and the 1000 Genome pilot project SNPs and indels. ${ }^{31}$ The vast majority of these variants $(899=93 \%)$ were located within the genomic regions with LOD scores below -2 and were excluded from further analysis. From the remaining 62 variants, 15 variants were predicted to have deleterious effects based on SIFT or Polyphen prediction software, ${ }^{32,33}$ from which 6 variants were proven to be false positive by Sanger sequencing, 3 variants were not located near a reported gene and, from the remaining 6 variants, only 3 were confirmed by Sanger sequencing to co-segregate with the phenotype within the pedigree: c.3647C > T (p.(Ser1216Leu); rs149524209) in Calmodulin-Regulated SpectrinAssociated Protein 1 (CAMSAP1, NM_015447.3); c.913A > G (p.(Ile305Val); rs139253452) in Nuclear-Interacting Partner of Anaplastic Lymphoma Kinase (ZC3HC1; NM_016478.3) and c.2066A>G (p.(Asp689Gly)) in MCF.2 Cell Line Derived Transforming Sequencelike Protein (MCF2L, NM_001112732.1). Next, we tested whether the three variants were present in different replication cohorts. The CAMSAP1 variant was found in 13 healthy volunteers of the Dutch Sanquin Blood Bank cohort (0.9\%) and the ZC3HC1 variant was found in 6 individuals of the Premature AtheroSclerosis cohort $(0.6 \%)$, in 3 healthy volunteers of the Sanquin Cohort $(0.2 \%)$ and in 5 volunteers of the Cambridge Biobank Resource $(0.06 \%$; Supplementary Table S4). Thus, both variants were eliminated for further studies. The MCF2L c.2066A > G; p.(Asp689Gly) variant was not found in any of the replication cohorts. MCF2L is located on chromosome 13q34 and harbors a highly conserved Rho-GEF DblHomology (DH) domain encoding the GEF activity specific for Rho family members and a pleckstrin homology domain $(\mathrm{PH})$ that binds inositol phosphates (Figure 1d). The c.2066G > A; p.(Asp689Gly) variant is located in a highly conserved region of the $\mathrm{DH}$ domain. MCF2L is a guanine-nucleotide exchange factor (GEF) from the Rho family that are signalling molecules of Rho proteins. MCF2L is

Table 1 Characteristics of the seven relatives used in exclusion linkage

\begin{tabular}{lcc}
\hline & No CVD & CVD \\
\hline MCF2L mutation carrier & 0 & $5^{\mathrm{a}}$ \\
Sex $(N=$ male/female) & $0 / 2$ & $3 / 2$ \\
Age & $61 /-$ & $59.4 \pm 4.6$ \\
Age CVD & $\mathrm{N} / \mathrm{A}$ & $46.0 \pm 9.4$ \\
Smoking $(\mathrm{N}=$ Yes $)$ & Yes/no & 2 \\
BMI $\left(\mathrm{kg} / \mathrm{m}^{2}\right)$ & $22 / 23$ & $24.2 \pm 1.6$ \\
Total cholesterol $(\mathrm{mmol} / \mathrm{l})$ & $5.9 / 4.2$ & $4.1(3.9-4.7)$ \\
HDL cholesterol $(\mathrm{mmol} / \mathrm{l})$ & $2.1 / 1.4$ & $1.3(1.2-1.5)$ \\
LDL cholesterol $(\mathrm{mmol} / \mathrm{l})$ & $3.6 / 2.2$ & $2.1(1.9-2.7)$ \\
Triglyceride $(\mathrm{mmol} / \mathrm{l})$ & $0.5 / 1.5$ & $1.0(0.8-2.4)$
\end{tabular}

Data are expressed as mean \pm standard deviation or as number $(N)$. Lipid values are expressed as median with interquartile range. Subjects were considered smokers if they were current smokers or when they quit smoking within the last 5 years. Abbreviations: BMI, body mass index; HDL, high density lipoprotein; LDL, low density lipoprotein; N/A, not applicable. apedigree member I-1 was not included as no clinical data were available. 
ubiquitously expressed in circulating blood cells. Total white blood cell $M C F 2 L$ mRNA expression in heterozygous carriers of the variant was lower as compared with the control (Supplementary Figure S2, $P<0.05)$. Noteworthy, mRNA expression of Ras-related C3 botulinum toxin substrate $1 R A C 1$ and Ras homolog gene family $R H O A$ was decreased, but this did not reach statistical significance (Supplementary Figure S2).

MCF2L689Gly variant is impaired in activating the Racl pathway As a GEF, MCF2L has been described to activate the small GTPases Racl and RhoA. ${ }^{34}$ We used a classical Rac1 pull-down assay to assess the ability of MCF2L to activate Rac1. HeLa cells were transfected with either wild-type MCF2L689Asp or mutant MCF2L689Gly and Rac1. GTP was measured (Figure 2). MCF2L expression resulted in the activation of Rac1, whereas MCF2L689Gly failed to activate Rac1. Next to Rac1, MCF2L may also activate RhoA, which is specifically involved in the formation of stress fibers by bundling the F-actin filaments. In the next experiment, we tested whether MCF2L expression resulted in RhoA activation by studying stress fiber formation. Indeed, the expression of wild-type MCF2L689Asp in $\mathrm{HeLa}$ cells resulted in increased stress fiber formation and cell rounding, both characteristics of RhoA activation (Figure 3). In contrast, MCF2L689Gly expression failed to induce the formation of stress fibers or cell rounding (Figure 3). Together, these experiments show that MCF2L689Gly is not involved in activating both Rac1 and RhoA.

\section{MCF2 $\mathrm{L}$ is present in atherosclerotic segments in humans}

MCF2L was profoundly detected in human coronary artery segments from patients with different grades of atherosclerosis (Figure 4a) but not in non-diseased arteries (Figure 4b). Human tonsil tissue served as a positive control (Supplementary Figure S3A). Staining without the secondary antibody in either atherosclerotic lesions or tonsil tissue was negative (Figure 4c and Supplementary Figure S3B). MCF2L did not co-localize with CD68, a marker for macrophages, CD34 (endothelial cell marker) or smooth muscle cells actin (Figure $4 \mathrm{~d}$ and f), but colocalized with antigen-presenting cells such as CD11c, CD3 type I helper cells and CD8 cytotoxic T cells within the atherosclerotic plaque (Figure $4 \mathrm{~g}$ and $\mathrm{i}$ ).

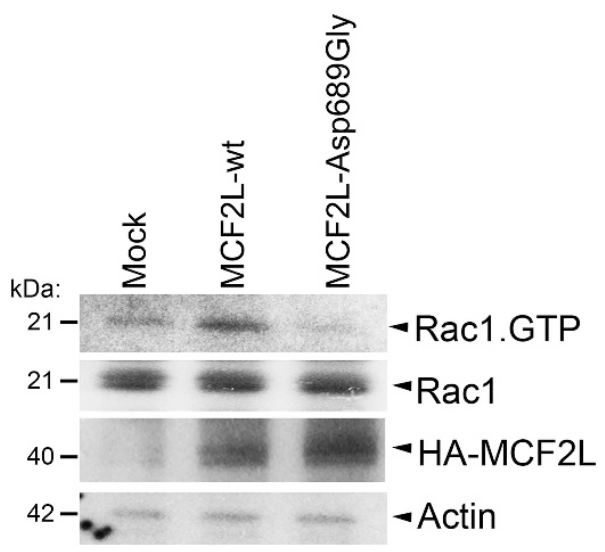

Figure 2 MCF2L689Gly does not activate Rac1. The MCF2L-689Gly variant does not activate Rac1. Hela cells were transiently transfected with wild-type MCF2L689Asp or the mutant MCF2L689Gly constructs. Rac1 protein was measured in cell lysates. Rac1-GTP was measured as described in methods section. Wild-type MCF2L689Asp but not MCF2L689Gly activates endogenous Rac1. Beta-actin was used as a loading control.

\section{DISCUSSION}

The aim of the current study was to identify the genetic defect underlying premature CVD in a small pedigree with an autosomal dominant form of CVD. We identified a rare variant in $M C F 2 L$ at position 689 where an aspartic acid was changed to a glycine. The variant was not present in 10376 healthy controls nor in 935 patients with premature CVD. The MCF2L c.2066A>G; p.(Asp689Gly) variant is located in the $\mathrm{DH}$ domain. Interestingly, MCF2L was absent in healthy artery segments, but abundantly detected in atherosclerotic lesions and co-localized with CD3 type I helper T cells and CD8 cytotoxic T cells.

MCF2L belongs to the Dbl family, which are guanine nucleotide exchange factors (GEFs) in particular Rho guanosine tri-phosphatase (Rho-GTPases). MCF2L contains a Dbl homology- (DH-), a pleckstrin homology- (PH-) domain and a CRAL-TRIO domain (Figure 1d). ${ }^{35}$ The DH domain is crucial for MCF2L function. The specific function of the PH-domain is largely unknown, but initial studies suggest a supportive function for the binding to the plasma membrane. ${ }^{34} \mathrm{MCF} 2 \mathrm{~L}$ provides the shift of GDP to GTP, resulting in the activation of Rho-GTPases. It is the MCF2L-DH domain that has been shown to play an important role in the interaction with and activation of the small GTPases RhoA, Racl and CDC42. ${ }^{36,37}$ RhoA functions as a molecular switch to regulate the assembly of actin stress fibers. ${ }^{38,39}$ Here, we showed in vitro that the MCF2L689Gly variant was defective in activating Racl and consequently no actin stress fibers were formed, which is highly indicative of impaired RhoA activation by the MCF2L variant. These defects may result in increased permeability and perturbed cell migration in the presence of the MCF2L689Gly variant. Noteworthy, abnormal activity of Rho-family GTPases has been reported in cardiovascular disorders. ${ }^{40}$ Endothelial dysfunction reflected by reduced vasodilation is a critical factor contributing to the pathogenesis of CVD.

GEFs together with Rho kinases play a pivotal role in leukocyte migration. ${ }^{41}$ The first step of leukocyte attachment involves rolling of the leukocytes over the endothelium mediated by different selectins. The subsequent clustering to adhesion molecules like ICAM-1 and VCAM-1 induces intracellular signalling of the endothelium involving RhoA and Racl activation. Although it is unclear whether abnormal RhoA or Rac1 activation, as observed in the presence of the MCF2L c.2066A > G; p.(Asp689Gly) variant, may interfere with leukocyte transendothelial migration, we observed that RAC1 and RHOA mRNA expressions in circulating blood cells was altered in $M C F 2 L$ c.2066A> G; p (Asp698Gly) carriers as compared with healthy controls. Furthermore, we observed increased MCF2L protein in atherosclerotic tissue segments that co-localized with CD3 and CD8, markers for $\mathrm{T}$ cells. Further studies are required to study to what extent circulating cell behavior is underlying the pathogenesis that is observed in this family.

The application of a combined exclusion linkage analysis and nextgeneration sequencing approach has been proven instrumental to identify novel mutations. ${ }^{21}$ In the past, owing to technical constraints, most of the pedigree studies have focused on the identification of molecular defects in pathways with an established role in atherosclerosis. This resulted in the identification of MEF2A and LRP6. ${ }^{7-10}$ Next-generation sequencing has provided us with an opportunity to sequence large numbers of genes in an unbiased approach without knowledge of the underlying biological process. This unbiased approach is likely to give a great impetus to our understanding of the pathology of complex diseases such as CVD. The identification of the novel variant in $M C F 2 L$ has proven that our approach is a successful method to identify rare variants in smaller families. 

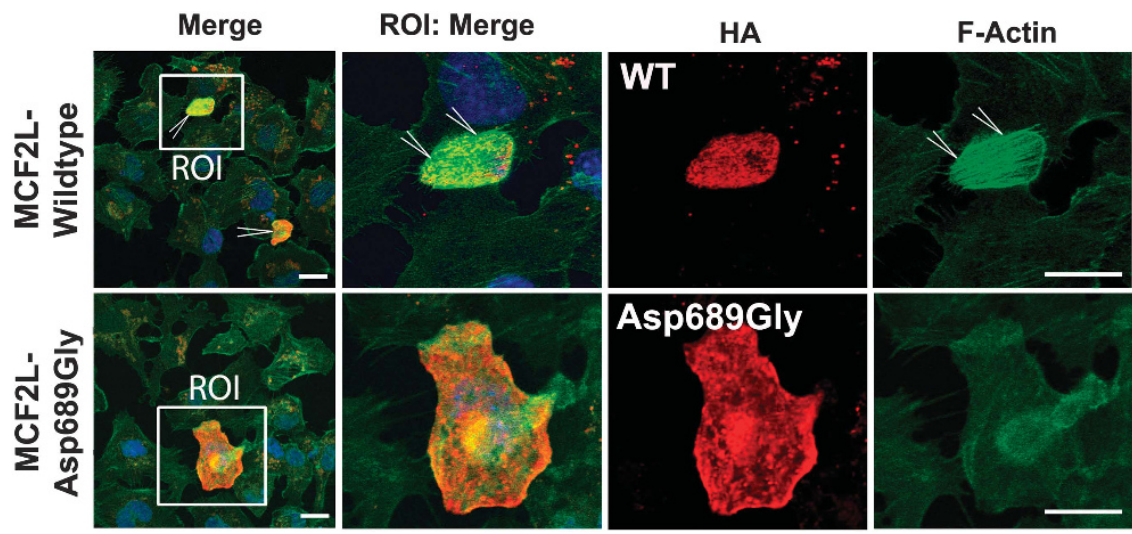

Figure 3 MCF2L689Asp but not MCF2L689Gly induces the RhoA phenotype. Wild-type MCF2L689Asp and MCF2L689Gly were expressed in HeLa cells Immunofluorescence imaging showed that wild-type MCF2L689Asp gives a RhoA phenotype with round cells and pronounced stress fibers (F-Actin, green, white square). MCF2L689Gly did not show an effect on the cell phenotype. The cells stay flat and do not show stress fibers (F-Actin green). F-Actin expression and wild-type MCF2L689Asp are co-localized as shown in the merged images (DAPI, blue). ROI, region of interest.
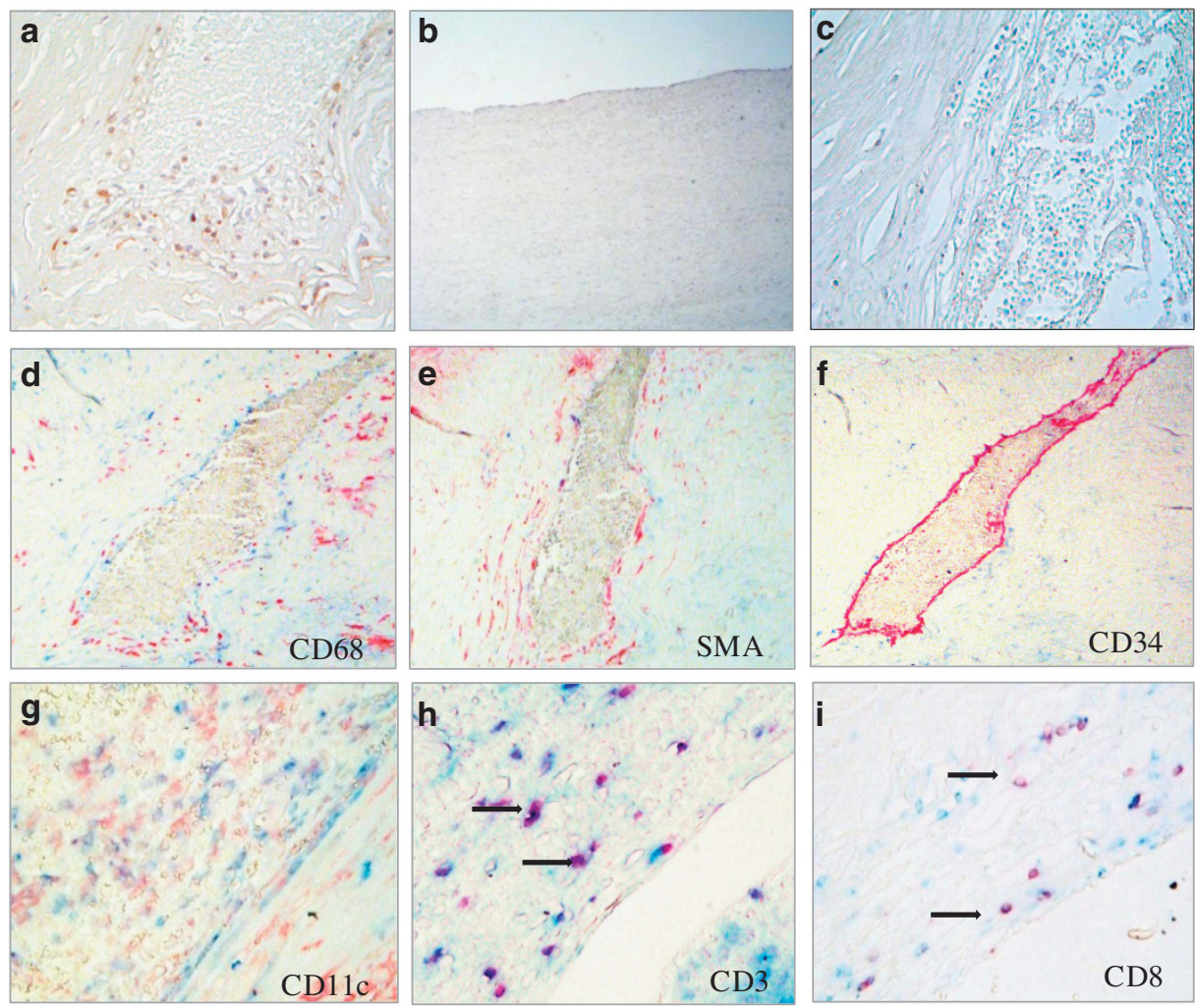

Figure $4 \mathrm{MCF} 2 \mathrm{~L}$ protein is detected in atherosclerotic but not in non-diseased coronary arterial segments and co-localized with CD3 and CD8 Cells. (a) Single staining of MCF2L (brown) in human atherosclerotic tissue. (b) Absence of MCF2L in a normal healthy coronary artery tissue segments. (c) Negative control in atherosclerotic tissue using only the secondary antibody. No background signal is observed. (d) No co-localization of MCF2L (blue) with CD68 (red). (e) No co-localization with smooth muscle alpha-actin (SMA red). (f) No co-localization with CD34 (red). (g) Co-localization of MCF2L (purple) with CD11c antigen-presenting cells, (h) with CD3 type I helper cells and (i) CD8 cytotoxic T cells.

\section{Limitations}

A major limitation of the current study is, however, that only circumstantial evidence for the role of this variant in the CVD phenotype could be gathered. With the use of exclusion linkage, we originally identified three rare variants in three different genes, but only one of them was uniquely present in the family. We did not identify this variant in other cases with premature atherosclerosis.
However, the presence of MCF2L protein in diseased tissue and the observation that the variant led to impaired MCF2L-DH-domaindependent actin stress fiber formation does suggest that MCF2L may play a role in the underlying pathobiology of premature atherosclerosis.

In conclusion, we have identified a functional variant in $M C F 2 L$ using exclusion linkage analysis and next-generation sequencing in a 
pedigree with premature CVD. Further studies are warranted to confirm our findings and to establish whether MCF2L may be an interesting target for therapy.

\section{CONFLICT OF INTEREST}

The authors declare no conflict of interest.

\section{ACKNOWLEDGEMENTS}

We would like to acknowledge all participants. We thank C. Holtkamp for the genetic fieldwork and J. Kuipers, C. Aalbers, S. Nurnberg and J. Peter for technical and laboratory assistance. We gratefully acknowledge the participation of all NIHR Cambridge BioResource volunteers. We thank the Cambridge BioResource staff for their help with volunteer recruitment. SM has been supported by the Marie-Curie NetSim ITN grant [grant number EC-215820] from the European Commission 7th Framework Program and Ipse Movet. SS is supported by a grant from ATHEROS. MMM was supported by Fondation LeDucq (Transatlantic Network, 2009-2014). JJPK is a recipient of the Lifetime Achievement Award of the Dutch Heart Foundation (2010T082). Research in WHO group is supported by program grants from the National Institute for Health Research (NIHR) (RP-PG-0310-1002), from the British Heart Foundation [RG/09/12/28096], from the European Commission and the Medical Research Council. The project made use of NHS Blood and Transplant donors from the Cambridge BioResource (http://www.cambridgebioresource. org.uk/). This local resource for genotype-phenotype association studies is supported by a grant from the NIHR to the Cambridge Biomedical Research Centre. GKH is holder of a Veni grant [91612122] from the Netherlands Organisation for Scientific Research (NWO). This work is supported by CardioVascular Research Initiative [CVON2011-19; Genius] and the European Union [Resolve: FP7-305707 and TransCard: FP7-603091-2].

1 Libby P, Ridker PM, Hansson GK: Progress and challenges in translating the biology of atherosclerosis. Nature 2011; 473: 317-325

2 Lee K, Sung J, Lee SC et al: Segment-specific carotid intima-media thickness and cardiovascular risk factors in Koreans: the Healthy Twin Study. Eur J Prev Cardiol 2012; 19: $1161-1172$

3 Sivapalaratnam S, Boekholdt SM, Trip MD et al: Family history of premature coronary heart disease and risk prediction in the EPIC-Norfolk prospective population study. Heart 2010; 96: 1985-1989.

4 Manolio TA, Collins FS, Cox NJ et al: Finding the missing heritability of complex diseases. Nature 2009; 461: 747-753.

5 Deloukas $\mathrm{P}$, Kanoni S, Willenborg $\mathrm{C}$ et al: Large-scale association analysis identifies new risk loci for coronary artery disease. Nat Genet 2013; 45: 25-33.

6 Roberts R, Chen L, Wells GA, Stewart AF: Recent success in the discovery of coronary artery disease genes. Can J Physiol Pharmacol 2011; 89: 609-615.

7 Mani A, Radhakrishnan J, Wang $\mathrm{H}$ et al: LRP6 mutation in a family with early coronary disease and metabolic risk factors. Science 2007; 315: 1278-1282.

8 Guella I, Rimoldi V, Asselta R et al: Association and functional analyses of MEF2A as a susceptibility gene for premature myocardial infarction and coronary artery disease. Circ Cardiovasc Genet 2009; 2: 165-172.

9 Keramati AR, Singh R, Lin A et al: Wild-type LRP6 inhibits, whereas atherosclerosislinked LRP6R611C increases PDGF-dependent vascular smooth muscle cell proliferation. Proc Natl Acad Sci USA 2011; 108: 1914-1918.

10 Lieb W, Mayer B, Konig IR et al: Lack of association between the MEF2A gene and myocardial infarction. Circulation 2008; 117: 185-191.

11 Wang L, Fan C, Topol SE, Topol EJ, Wang Q: Mutation of MEF2A in an inherited disorder with features of coronary artery disease. Science 2003; 302: 1578-1581.

12 Austin MA, Hutter CM, Zimmern RL, Humphries SE: Familial hypercholesterolemia and coronary heart disease: a HuGE association review. Am J Epidemiol 2004; 160: 421-429.

13 Huijgen R, Vissers MN, Defesche JC, Lansberg PJ, Kastelein JJ, Hutten BA: Familial hypercholesterolemia: current treatment and advances in management. Expert Rev Cardiovasc Ther 2008; 6: 567-581.
14 Maiwald S, Sivapalaratnam S, Motazacker MM et al: Mutation in KERA identified by linkage analysis and targeted resequencing in a pedigree with premature atherosclerosis. PLoS One 2014; 9: e98289.

15 Erdmann J, Stark K, Esslinger UB et al: Dysfunctional nitric oxide signalling increases risk of myocardial infarction. Nature 2013; 504: 432-436.

16 Greenland P, Alpert JS, Beller GA et al: 2010 ACCF/AHA guideline for assessment of cardiovascular risk in asymptomatic adults: executive summary: a report of the American College of Cardiology Foundation/American Heart Association Task Force on Practice Guidelines. Circulation 2010; 122: 2748-2764.

17 Albers CA, Cvejic A, Favier R et al: Exome sequencing identifies NBEAL2 as the causative gene for gray platelet syndrome. Nat Genet 2011; 43: 735-737.

18 Abecasis GR, Cherny SS, Cookson WO, Cardon LR: GRR: graphical representation of relationship errors. Bioinformatics 2001; 17: 742-743.

19 O'Connell JR, Weeks DE: PedCheck: a program for identification of genotype incompatibilities in linkage analysis. Am J Hum Genet 1998; 63: 259-266.

20 Gudbjartsson DF, Jonasson K, Frigge ML, Kong A: Allegro, a new computer program for multipoint linkage analysis. Nat Genet 2000; 25: 12-13.

21 Motazacker MM, Pirruccello J, Huijgen R et al: Advances in genetics show the need for extending screening strategies for autosomal dominant hypercholesterolaemia. Eur Heart J 2012; 33: 1360-1366.

22 Surendran RP, Visser ME, Heemelaar S et al: Mutations in LPL, APOC2, APOA5, GPIHBP1 and LMF1 in patients with severe hypertriglyceridaemia. J Intern Med 2012; 272: 185-196.

23 Hauser ER, Mooser V, Crossman DC et al: Design of the Genetics of Early Onset Cardiovascular Disease (GENECARD) study. Am Heart J 2003; 145: 602-613.

24 Vergeer M, Boekholdt SM, Sandhu MS et al: Genetic variation at the phospholipid transfer protein locus affects its activity and high-density lipoprotein size and is a novel marker of cardiovascular disease susceptibility. Circulation 2010; 122: 470-477.

25 Dendrou CA, Plagnol V, Fung $\mathrm{E}$ et al: Cell-specific protein phenotypes for the autoimmune locus IL2RA using a genotype-selectable human bioresource. Nat Genet 2009; 41: 1011-1015.

26 Salerno JC, Jones RJ, Erdogan E, Smith SM: A single-stage polymerase-based protocol for the introduction of deletions and insertions without subcloning. Mol Biotechnol 2005: 29: 225-232.

27 Nethe M, Anthony EC, Fernandez-Borja $M$ et al: Focal-adhesion targeting links caveolin-1 to a Rac1-degradation pathway. J Cell Sci 2010; 123: 1948-1958.

28 van Rijssel J, Hoogenboezem M, Wester L, Hordijk PL, Van Buul JD: The N-terminal $\mathrm{DH}-\mathrm{PH}$ domain of Trio induces cell spreading and migration by regulating lamellipodia dynamics in a Rac1-dependent fashion. PLoS One 2012; 7: e29912.

29 van Buul JD, Voermans C, van den Berg V et al: Migration of human hematopoietic progenitor cells across bone marrow endothelium is regulated by vascular endothelial cadherin. J Immunol 2002; 168: 588-596.

$30 \mathrm{Ng}$ SB, Turner EH, Robertson PD et al: Targeted capture and massively parallel sequencing of 12 human exomes. Nature 2009; 461: 272-276.

31 Genomes Project C, Abecasis GR, Altshuler D et al: A map of human genome variation from population-scale sequencing. Nature 2010; 467: 1061-1073

32 Kumar P, Henikoff S, Ng PC: Predicting the effects of coding non-synonymous variants on protein function using the SIFT algorithm. Nat Protoc 2009; 4: 1073-1081.

33 Adzhubei IA, Schmidt S, Peshkin L et al: A method and server for predicting damaging missense mutations. Nat Methods 2010; 7: 248-249.

34 Rossman KL, Der CJ, Sondek J: GEF means go: turning on RHO GTPases with guanine nucleotide-exchange factors. Nat Rev Mol Cell Biol 2005; 6: 167-180.

35 Cheng L, Mahon GM, Kostenko EV, Whitehead IP: Pleckstrin homology domainmediated activation of the rho-specific guanine nucleotide exchange factor Dbs by Rac1. J Biol Chem 2004; 279: 12786-12793.

36 Ueda S, Kataoka T, Satoh T: Role of the Sec14-like domain of Dbl family exchange factors in the regulation of Rho family GTPases in different subcellular sites. Cell Signal 2004; 16: 899-906.

37 Rossman KL, Worthylake DK, Snyder JT, Siderovski DP, Campbell SL, Sondek J: A crystallographic view of interactions between Dbs and Cdc42: PH domain-assisted guanine nucleotide exchange. EMBO J 2002; 21: 1315-1326.

38 Ridley AJ, Hall A: The small GTP-binding protein rho regulates the assembly of focal adhesions and actin stress fibers in response to growth factors. Cell 1992; 70: 389-399.

39 Noma K, Kihara Y, Higashi Y: Striking crosstalk of ROCK signaling with endothelial function. J Cardiol 2012; 60: 1-6.

40 Rolfe BE, Worth NF, World CJ et al: Rho and vascular disease. Atherosclerosis 2005; 183: $1-16$.

41 Fernandez-Borja M, van Buul JD, Hordijk PL: The regulation of leucocyte transendothelial migration by endothelial signalling events. Cardiovasc Res 2010; 86: 202-210.

Supplementary Information accompanies this paper on European Journal of Human Genetics website (http://www.nature.com/ejhg) 